\title{
STEADY STATES IN A STRUCTURED EPIDEMIC MODEL WITH WENTZELL BOUNDARY CONDITION
}

\author{
ÀNGEL CALSINA AND JÓZSEF Z. FARKAS
}

\begin{abstract}
We introduce a nonlinear structured population model with diffusion in the state space. Individuals are structured with respect to a continuous variable which represents a pathogen load. The class of uninfected individuals constitutes a special compartment that carries mass, hence the model is equipped with generalized Wentzell (or dynamic) boundary conditions. Our model is intended to describe the spread of infection of a vertically transmitted disease, for example Wolbachia in a mosquito population. Therefore the (infinite dimensional) nonlinearity arises in the recruitment term. First we establish global existence of solutions and the Principle of Linearised Stability for our model. Then, in our main result, we formulate simple conditions, which guarantee the existence of non-trivial steady states of the model. Our method utilizes an operator theoretic framework combined with a fixed point approach. Finally, in the last section we establish a sufficient condition for the local asymptotic stability of the positive steady state.
\end{abstract}

\section{IntRoduction OF THE MODEL}

Structured population dynamics is an exciting field of research in mathematical biology, see e.g. [12, 29, 32, 38] for a collection of classical models and results in the area. Structured population models often assume spatial homogeneity of the population in a given habitat and only focus on the dynamics of the population arising from differences between individuals with respect to some physiological structure. In a general model, reproduction, death and growth characterize individual behaviour, which may be affected by competition (scramble or contest), for example for available resources or for a mating partner. One usually incorporates certain types of nonlinearities via density dependence in the vital rates to account for these biological phenomena.

Traditionally, structured population models have been formulated as first order nonlinear partial differential equations of hyperbolic type and therefore the analysis of these models is often challenging from the mathematical point of view. In the very recent paper [27] Hadeler introduced size-structured population models with diffusion in the

Date: September 18, 2012.

1991 Mathematics Subject Classification. 92D25, 47N60, 47D06, 35B35.

Key words and phrases. Structured populations; diffusion; Wentzell-Robin boundary condition; steady states; spectral methods;. 
size-space. The biological motivation is that diffusion allows for "stochastic noise" to be incorporated in the model equations in a deterministic fashion. In [27] Hadeler mainly addressed the question that what type of boundary conditions are necessary to be imposed for a biologically plausible and mathematically sound model. In this context particular cases of a Robin boundary condition were considered. We note that other researchers also investigated recently the effects of introducing stochasticity (for example variation in individual growth rates) via diffusion in structured population models, see $[3,4,9]$.

In this paper we introduce and analyze a structured population model, with so called distributed recruitment term and generalized Wentzell boundary conditions. Our model intends to describe the dynamics of a population, which is infected with a certain type of bacteria, see later for more details. At the same time our model is general enough to make the forthcoming analysis to be applicable for similar classes of models. In particular, we consider the following model:

$$
\begin{aligned}
u_{t}(x, t)+(\gamma(x) u(x, t))_{x} & =\left(d(x) u_{x}(x, t)\right)_{x}-\mu(x) u(x, t) \\
& +\beta_{0}(U(t)) \int_{0}^{m} \beta_{1}(x, y) \beta_{2}\left(\frac{\int_{y}^{m} u(r, t) \mathrm{d} r}{U(t)}\right) u(y, t) \mathrm{d} y, x \in(0, m),
\end{aligned}
$$

$$
\begin{aligned}
& {\left[\left(d(x) u_{x}(x, t)\right)_{x}\right]_{x=0}-(d(0)+\gamma(0)) u_{x}(0, t)} \\
& \quad+\left(\gamma(0)-\gamma^{\prime}(0)-b_{0}(U(t)) b_{2}\left(\frac{\int_{0}^{m} u(r, t) \mathrm{d} r}{U(t)}\right)\right) u(0, t)=0, \\
& d(m) u_{x}(m, t)-\gamma(m) u(m, t)=0,
\end{aligned}
$$

with a suitable initial condition. The function $u=u(x, t)$ denotes the density of individuals of pathogen load $x$ at time $t$. This means that the total number of individuals with pathogen (or bacterium) load between $x_{1}$ and $x_{2}$ is given by $\int_{x_{1}}^{x_{2}} u(y, t) \mathrm{d} y$. It is then clear that the uninfected individuals constitute a separate special class. Therefore, in contrast to any other state, the state $x=0$ carries mass. Hence we specify the value of the function $u$ at $x=0$, which gives the total number of individuals in the uninfected class. Then the total population size at any time $t$ is given by

$$
U(t)=|u(0, t)|+\int_{0}^{m}|u(r, t)| \mathrm{d} r=\|\mathbf{u}\|_{\mathcal{X}}
$$

In mathematical terms, we distinguish between equivalence classes of the state space $L^{1}$, which are represented by functions, which have different values at $x=0$. Or, in other words, to each equivalence class of the Lebesgue space $L^{1}$ we assign a real number, which is its "value" at $x=0$. This is expressed in the Wentzell-type boundary condition (1.2). We also assume a maximal infection load $m$. The function $\gamma$ represents the reproduction rate of the bacterium in the host. Since the maximum infection load is $m$ we have the boundary condition (1.3), i.e. the infection load cannot increase above $m$ in any individual in the population. It is shown by a straightforward calculation (see 
e.g. [17] for a similar calculation) that the boundary conditions (1.2)-(1.3) guarantee conservation of the total population size in the absence of mortality and recruitment. As usual, $\mu$ denotes the mortality rate (both natural mortality and extra mortality due to the infection) of individuals, while $d$ stands for diffusion. Now we impose the following general assumptions on the model ingredients:

$$
\begin{aligned}
& \beta_{0}, b_{0} \in C([0, \infty)), \beta_{2}, b_{2} \in C([-1,1]), \mu \in C([0, m]), \quad \beta_{1} \in C([0, m] \times[0, m]), \\
& \beta_{0}, \beta_{1}, \beta_{2}, b_{0}, b_{2}, \mu \geq 0, \quad \gamma, d \in C^{1}([0, m]), \quad d>0 .
\end{aligned}
$$

In addition we will make some technical assumptions on some of the model ingredients later on. Our goal is to describe a model of a population, which is infected with a disease that is only transmitted vertically; hence the only nonlinearity arises in the term specifying the recruitment of individuals. To be more precise, we are interested in the dynamics of Wolbachia infections in mosquito populations. Wolbachia is a maternally transmitted endosymbiont bacterium, which infects around $70 \%$ of the arthropod species. Recent laboratory results suggest, see [31], that a stable introduction of a particular strain of Wolbachia into A. Aegypti halved the average life span of an adult mosquito. This represents a potential tool to eradicate mosquito born diseases (like malaria, dengue or West Nile virus), since the life span of the mosquito is reduced to 2-3 weeks which more or less equals the intrinsic excubation period of the disease, i.e. the time needed for a mosquito to become infectious after feeding on an infected human. According to [31], high maternal inheritance, strong cytoplasmic incompatibility and low costs to reproductive output are the key factors for prevalence of the Wolbachia infection. Cytoplasmic incompatibility (CI for short) means that from a mating of an uninfected female and infected male there is no (complete CI) or only a very few (partial CI) number of viable offspring. We refer the interested reader to $[14,16,34]$ for more biological background and mathematical modelling of populations infected with a cytoplasmic incompatibility inducing Wolbachia. The possible underlying mechanisms for complete or partial expression of CI are still a matter of debate for different species. In our model we take the following view of partial CI, see $[6,25]$ for further reference. We make the assumption that a female who has bacterium load $y$ can successfully mate only with males of bacterium load less than $y$. Therefore the function $\beta_{2}$, which determines the fertility rate of an individual with infection load $y$, depends on the proportion of the individuals, which have infection load higher than $y$. In particular $\beta_{2}$ is a monotone decreasing function of its argument. In other words, we assume that the sex ratio is $1: 1$ and the probability for a female mosquito of producing a viable offspring is a monotone decreasing function of the proportion of the male population size that have higher infection load. The function $\beta_{0}$ represents competition effects due for example to limitations in available resources. Similarly, $b_{2}$ measures the reproductive success of uninfected females and $b_{0}$ represents the effects of competition on the reproduction of uninfected individuals. The maternal transmission rate is determined by the function $\beta_{1}$, i.e. infected mothers with bacterium load $y$ give birth to offspring with bacterium load $x$ at a rate of $\beta_{1}(x, y)$. 
The boundary condition (1.2) can be rewritten, and more easily understood from the point of view of its biological meaning, in the dynamic form (by formally replacing the diffusion operator from equation (1.1) on the boundary) as:

$$
\begin{aligned}
u_{t}(0, t) & =u(0, t)\left(-\gamma(0)-\mu(0)+b_{0}(U(t)) b_{2}\left(\frac{\int_{0}^{m} u(r, t) \mathrm{d} r}{U(t)}\right)\right) \\
& +u_{x}(0, t) d(0)+\beta_{0}(U(t)) \int_{0}^{m} \beta_{1}(0, y) \beta_{2}\left(\frac{\int_{y}^{m} u(r, t) \mathrm{d} r}{U(t)}\right) u(y, t) \mathrm{d} y .
\end{aligned}
$$

It is not difficult to verify that in the absence of mortality and recruitment, i.e. taking $\mu \equiv 0$ and $b_{0} \equiv \beta_{0} \equiv 0$, the total population size $U(t)$ is conserved. Notice that in this case, $u_{t}(0, t)$ equals $-\gamma(0) u(0, t)+d(0) u_{x}(0, t)$, i.e., the flux through $x=0$ due to reproduction of the bacteria (from the biological point of view it would be natural to assume $\gamma(0)=0$, but we do not impose such a restriction here) and to diffusion (noise) in the second term (this could take into account a certain -very weak- horizontal transmission).

Our main goal in this paper is to establish sufficient conditions for the existence of positive steady state solutions of model (1.1)-(1.3). We shall refer the interested reader to $[5,8,15,17]$ where different size-structured models with distributed recruitment processes were investigated. The boundary condition (1.2) is the so called generalized Wentzell-Robin or dynamic boundary condition. These "unusual" boundary conditions were investigated recently for models describing physical processes such as diffusion and wave propagation, see e.g. $[19,20,26]$. Briefly, they are used to model processes where particles reaching the boundary of a domain can be either reflected from the boundary or they can be absorbed and then released after some time. Recently in [17] we introduced, as far as we know for the first time, Wentzell-type boundary conditions in the context of physiologically structured populations with a distributed recruitment process and with diffusion in the size space. The introduction of diffusion in the size space is very natural from the application point of view, since in the real world individuals who start their life in the same cohort do not finish their life so, due for example to stochastic variations in individual growth rates. We refer the reader to [27] (see also [9]) where different types of population models were introduced with diffusion in the size-space.

The idea of considering population models where the structuring variable represents a pathogen load is clearly not new. In fact in [37] Waldstädter et al. introduced a similar model, where they have derived a dynamic boundary condition for the special class of uninfected individuals. In their model this class however does not correspond to the value $u(0, t)$, in fact the uninfected population size is represented by a new variable $U(t)$ and a transition condition between the compartment $U$ and $u(0, t)$ is given. This implies two things: firstly $u(0, t) \neq U(t)$, secondly individuals who are sitting in the special compartment $U$ are not subject to diffusion, i.e. first they need to enter the state $u(0, t)$. This in some sense may seem counterintuitive, as diffusion is incorporated to 
model stochastic noise, which could result in low probability random infections. Since we model an (almost) completely vertically transmitted disease, we do not take into account infection in a usual way. By usual way we mean by means of a nonlinear transition rate of the type $S I$, which in our model corresponds to $u(0, t) \times \int_{0}^{m} u(x, t) \mathrm{d} x$. But, due to the nature of the Wentzell boundary condition we allow transition between the uninfected and infected compartments as a diffusive flux through $x=0$ in both directions (one of them corresponding to random infections and the other one to spontaneous, i.e. random healings), depending on the sign of $u_{x}$, as commented above. We note that the mathematical analysis presented in [37] is carried out in the Hilbert space $L^{2}$.

We shall mention here that the first papers introducing boundary conditions that involve second order derivatives for parabolic or elliptic differential operators go back to the 1950s, see the papers by Feller [22, 23] and Wentzell (also transliterated as "Ventcel' ", Aleksandr D. Ventcelь) $[35,36]$. These first studies were purely motivated from the mathematical point of view. The original question, as far as we know, was to identify the maximal set of possible boundary conditions that give rise for a parabolic differential operator to generate a contraction semigroup on an appropriate state space.

In this paper first we establish global existence and positivity of solutions of model (1.1)-(1.3). Our existence proof relies on the existence of the semigroup governing the linear part of the model, which was established in [17] (with two point Wentzell boundary conditions) following similar arguments developed in [19,20], and on the analyticity of this linear semigroup, which was very recently established in [21] (and in fact for higher dimensional domains, as well). Then in Section 3 we investigate the existence of non-trivial steady states to our model. To treat the steady state problem, we devise Schauder's fixed point theorem combined with an operator theoretic approach. A similar operator theoretic framework was previously utilised for simpler problems (in particular with finite dimensional nonlinearities and classical boundary conditions), see e.g. [5, 15]. The key idea to treat the steady state problem is to define a linear operator for a fixed environment (nonlinearity) and to study spectral properties of that operator. Finally in the last section we establish some sufficient stability conditions for the positive steady state.

\section{EXISTENCE AND POSITIVITY OF SOLUTiOnS}

In this section we establish global existence (and positivity) of solutions of the nonlinear problem (1.1)-(1.3). Throughout the section we employ some standard results from [28]. We introduce the state space $\mathcal{X}=L^{1}(0, m) \oplus \mathbb{R}$ with norm

$$
\|\mathbf{u}\|=\left\|\left(u, u_{0}\right)\right\|=\|u\|_{1}+\left|u_{0}\right|
$$

which is a Banach lattice. Next we write our problem (1.1)-(1.3) in the form of an abstract Cauchy problem:

$$
\frac{\mathrm{d} \mathbf{u}}{\mathrm{d} t}-A \mathbf{u}=F(\mathbf{u}), \quad t>0, \quad \mathbf{u}(0)=\mathbf{u}_{0}
$$


where $A$ is the linear operator defined by

$$
A \mathbf{u}=\left(\begin{array}{c}
\mathcal{A} u \\
-\mu(0) u(0)+d(0) u^{\prime}(0)-\gamma(0) u(0)
\end{array}\right) .
$$

In (2.6) the operator $\mathcal{A}$ is defined as

$$
\mathcal{A} u=\frac{\partial}{\partial x}\left(d(\cdot) \frac{\partial u}{\partial x}\right)-\frac{\partial}{\partial x}(\gamma(\cdot) u)-\mu(\cdot) u
$$

The domain of the linear part $A$ is given by

$$
\begin{aligned}
D(A)=\{ & \left\{u \in C^{2}[0, m]: \mathcal{A} u \in L^{1}(0, m), \quad d(m) u^{\prime}(m)-\gamma(m) u(m)=0\right. \\
& \left.\left.\left(d(s) u^{\prime}(s)\right)^{\prime}\right|_{s=0}-(d(0)+\gamma(0)) u^{\prime}(0)+\left(\gamma(0)-\gamma^{\prime}(0)\right) u(0)=0\right\} .
\end{aligned}
$$

The nonlinear but bounded function $F$ is defined as

$$
F(\mathbf{u})=\left\{\begin{array}{rll}
F^{0}(\mathbf{u}) & \text { if } & \mathbf{u} \in \mathcal{X} \backslash\{\mathbf{0}\} \\
\mathbf{0} & \text { if } & \mathbf{u}=\mathbf{0}
\end{array}\right\}
$$

with $D(F)=\mathcal{X}$, where

$$
F^{0}(\mathbf{u})=\left(\begin{array}{c}
\beta_{0}(\|\mathbf{u}\|) \int_{0}^{m} \beta_{1}(\cdot, y) \beta_{2}\left(\frac{\int_{y}^{m} u(r) \mathrm{d} r}{\|\mathbf{u}\|}\right) u(y) \mathrm{d} y \\
\beta_{0}(\|\mathbf{u}\|) \int_{0}^{m} \beta_{1}(0, y) \beta_{2}\left(\frac{\int_{y}^{m} u(r) \mathrm{d} r}{\|\mathbf{u}\|}\right) u(y) \mathrm{d} y+b_{0}(\|\mathbf{u}\|) b_{2}\left(\frac{\int_{0}^{m} u(y) \mathrm{d} y}{\|\mathbf{u}\|}\right) u_{0}
\end{array}\right) .
$$

Note that $F^{0}$ is only defined on the open set $\mathcal{X} \backslash\{\mathbf{0}\}$.

Theorem 2.1. Assume that $\beta_{0}, \beta_{2}, b_{0}$ and $b_{2}$ are locally Lipschitzian. Then a unique solution of problem (2.5) exists for all positive times for any $u_{0} \in \mathcal{X}$.

Proof. We use [28, Corollary 3.3.5] to establish global existence of solutions to the semilinear problem (2.5). To this end, first we show that $F$ is locally Lipschitz continuous, i.e. for every $\mathbf{u} \in \mathcal{X}$ there exists a neighbourhood $U \subset \mathcal{X}$ of $\mathbf{u}$ such that for every $\mathbf{u}_{1}, \mathbf{u}_{2} \in U$ we have $\left\|F\left(\mathbf{u}_{1}\right)-\left.F\left(\mathbf{u}_{2}\right)\right|_{\mathcal{X}} \leq L\right\| \mathbf{u}_{1}-\mathbf{u}_{2} \|_{\mathcal{X}}=L\left(\left\|u_{1}-u_{2}\right\|_{1}+\left|u_{1_{0}}-u_{2_{0}}\right|\right)$ for some constant $L \geq 0$.

If either $\mathbf{u}_{1}=\mathbf{0}$ or $\mathbf{u}_{2}=\mathbf{0}$ then since $F$ is bounded (i.e. all the ingredients $\beta_{0}, \beta_{1}, \beta_{2}, b_{0}$ and $b_{2}$ are continuous, hence bounded) and $F(\mathbf{0})=\mathbf{0}$ we have the Lipschitz property 
$\|F(\mathbf{u})\| \leq L\|\mathbf{u}\|$ for some $L>0$. For $\mathbf{u}_{1} \neq \mathbf{0}, \mathbf{u}_{2} \neq \mathbf{0}$ we have:

$$
\begin{aligned}
& \left.\left\|F\left(\mathbf{u}_{1}\right)-F\left(\mathbf{u}_{2}\right)\right\|\right|_{\mathcal{X}} \leq \\
& \left|\beta_{0}\left(\left\|\mathbf{u}_{1}\right\|\right)-\beta_{0}\left(\left\|\mathbf{u}_{2}\right\|\right)\right|\left\|\int_{0}^{m} \beta_{1}(\cdot, y) \beta_{2}\left(\frac{\int_{y}^{m} u_{1}(r) \mathrm{d} r}{\left\|\mathbf{u}_{1}\right\|}\right) u_{1}(y) \mathrm{d} y\right\|_{1} \\
& +\beta_{0}\left(\left\|\mathbf{u}_{2}\right\|\right)\left\|\int_{0}^{m} \beta_{1}(\cdot, y)\left(\beta_{2}\left(\frac{\int_{y}^{m} u_{1}(r) \mathrm{d} r}{\left\|\mathbf{u}_{1}\right\|}\right) u_{1}(y)-\beta_{2}\left(\frac{\int_{y}^{m} u_{2}(r) \mathrm{d} r}{\left\|\mathbf{u}_{2}\right\|}\right) u_{2}(y)\right) \mathrm{d} y\right\| \\
& +\left|\beta_{0}\left(\left\|\mathbf{u}_{1}\right\|\right)-\beta_{0}\left(\left\|\mathbf{u}_{2}\right\|\right)\right|\left|\int_{0}^{m} \beta_{1}(0, y) \beta_{2}\left(\frac{\int_{y}^{m} u_{1}(r) \mathrm{d} r}{\left\|\mathbf{u}_{1}\right\|}\right) u_{1}(y) \mathrm{d} y\right| \\
& +\beta_{0}\left(\left\|\mathbf{u}_{2}\right\|\right)\left|\int_{0}^{m} \beta_{1}(0, y)\left(\beta_{2}\left(\frac{\int_{y}^{m} u_{1}(r) \mathrm{d} r}{\left\|\mathbf{u}_{1}\right\|}\right) u_{1}(y)-\beta_{2}\left(\frac{\int_{y}^{m} u_{2}(r) \mathrm{d} r}{\left\|\mathbf{u}_{2}\right\|}\right) u_{2}(y)\right) \mathrm{d} y\right| \\
& +\left|b_{0}\left(\left\|\mathbf{u}_{1}\right\|\right)-b_{0}\left(\left\|\mathbf{u}_{2}\right\|\right)\right| b_{2}\left(\frac{\int_{0}^{m} u_{1}(r) \mathrm{d} r}{\left\|\mathbf{u}_{1}\right\|}\right) u_{1_{0}} \\
& +b_{0}\left(\left\|\mathbf{u}_{2}\right\|\right)\left(b_{2}\left(\frac{\int_{0}^{m} u_{1}(r) \mathrm{d} r}{\left\|\mathbf{u}_{1}\right\|}\right) u_{1_{0}}-b_{2}\left(\frac{\int_{0}^{m} u_{2}(r) \mathrm{d} r}{\left\|\mathbf{u}_{2}\right\|}\right) u_{2_{0}}\right) .
\end{aligned}
$$

The term in (2.9) can be bounded above by $L_{\beta_{0}}|| \mathbf{u}_{1}-\mathbf{u}_{2}|| M_{\beta_{1}} M_{\beta_{2}} m\left\|u_{1}\right\|_{1}$, where $\beta_{0}<$ $M_{\beta_{0}}, \beta_{1}<M_{\beta_{1}}, \beta_{2}<M_{\beta_{2}}$ and $L_{\beta_{0}}$ is the Lipschitz constant of $\beta_{0}$ in $\left[0, \sup _{u \in U}\|u\|\right]$. To obtain the estimate for $(2.10)$ we note that

$$
\begin{aligned}
& \left|\beta_{2}\left(\frac{\int_{y}^{m} u_{1}(r) \mathrm{d} r}{\left\|\mathbf{u}_{1}\right\|}\right) u_{1}(y)-\beta_{2}\left(\frac{\int_{y}^{m} u_{2}(r) \mathrm{d} r}{\left\|\mathbf{u}_{2}\right\|}\right) u_{2}(y)\right| \\
& =\left|\left(\beta_{2}\left(\frac{\int_{y}^{m} u_{1}(r) \mathrm{d} r}{\left\|\mathbf{u}_{1}\right\|}\right)-\beta_{2}\left(\frac{\int_{y}^{m} u_{2}(r) \mathrm{d} r}{\left\|\mathbf{u}_{2}\right\|}\right)\right) u_{1}(y)+\beta_{2}\left(\frac{\int_{y}^{m} u_{2}(r) \mathrm{d} r}{\left\|\mathbf{u}_{2}\right\|}\right)\left(u_{1}(y)-u_{2}(y)\right)\right| \\
& \leq L_{\beta_{2}}\left\|\frac{\int_{y}^{m} u_{1}(r) \mathrm{d} r}{\left\|\mathbf{u}_{1}\right\|}-\frac{\int_{y}^{m} u_{2}(r) \mathrm{d} r}{\left\|\mathbf{u}_{1}\right\|}+\frac{\int_{y}^{m} u_{2}(r) \mathrm{d} r}{\left\|\mathbf{u}_{1}\right\|}-\frac{\int_{y}^{m} u_{2}(r) \mathrm{d} r}{\left\|\mathbf{u}_{2}\right\|}\right\| \|_{1}\left|u_{1}(y)\right|+M_{\beta_{2}}\left|u_{1}(y)-u_{2}(y)\right| \\
& \leq L_{\beta_{2}}\left(\left.\left\|\int_{y}^{m} u_{1}(r) \mathrm{d} r-\int_{y}^{m} u_{2}(r) \mathrm{d} r\right\|\right|_{1} \frac{\left|u_{1}(y)\right|}{\left\|\mathbf{u}_{1}\right\|}\right)+M_{\beta_{2}}\left|u_{1}(y)-u_{2}(y)\right|
\end{aligned}
$$

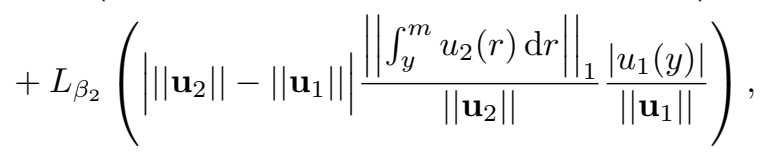


where $L_{\beta_{2}}$ is the Lipschitz constant of $\beta_{2}$ in $[-1,1]$. Similarly to (2.15), we obtain the appropriate estimates for the terms (2.11)-(2.14). Hence $F$ is locally Lipschitzian. Since $\|F(\mathbf{u})-F(\mathbf{0})\|=\|F(\mathbf{u})\| \leq L\|\mathbf{u}\|, F$ is also sublinear and since $A$ is sectorial (see [21]) the statement of Theorem 2.1 follows from [28, Corollary 3.3.5].

Note that $F: \mathcal{X}_{+} \rightarrow \mathcal{X}_{+}$. The solution of the Cauchy problem (2.5) can be written as

$$
\mathbf{u}(t)=\mathcal{T}(t) \mathbf{u}_{0}+\int_{0}^{t} \mathcal{T}(t-s) F(\mathbf{u}(s)) \mathrm{d} s, \quad t \in\left(0, t_{0}\right),
$$

where $\mathcal{T}$ is the linear semigroup generated by the closure of the sectorial operator $A$. We refer the reader to [17] were it is showed that a similar linear problem (with two point Wentzell boundary condition) is governed by a quasicontractive positive semigroup. Furthermore we refer to [21] where the sectoriality of the operator $A$ was shown in $L^{p}, 1 \leq p \leq \infty$, in general. Since $F$ is a positive operator and the semigroup $\mathcal{T}(t)$ is positive the variation formula (2.16) immediately shows positivity of solutions.

The Principle of Linearised Stability can be established for model (1.1)-(1.3) by directly applying again results from [28]. In particular, if $\mathbf{u}_{*}$ is an equilibrium point then let $F_{\mathbf{u}_{*}}^{\prime}$ denote the linearisation of $F$ at the equilibrium $\mathbf{u}_{*}$, which is a bounded linear operator defined on $\mathcal{X}$ (if it exists). See later in Section 4 for more details. Then $[28$, Theorem 5.1.1] implies that if $A$ is sectorial and $F$ is locally Lipschitz continuous then the equilibrium $\mathbf{u}_{*}$ is asymptotically stable if $\sigma\left(A+F_{\mathbf{u}_{*}}^{\prime}\right) \subset\{\lambda \in \mathbb{C} \mid \operatorname{Re}(\lambda)<\alpha<0\}$. On the other hand, [28, Theorem 5.1.3] implies (under the same conditions on $A$ and $F$ ) that the equilibrium $\mathbf{u}_{*}$ is unstable if $\sigma\left(A+F_{\mathbf{u}_{*}}^{\prime}\right) \cap\{\lambda \in \mathbb{C} \mid \operatorname{Re}(\lambda)>0\}$ is not empty.

\section{EXISTENCE OF NON-TRIVIAL STEADY STATES}

It is obvious that model (1.1)-(1.3) admits the trivial steady state. It is also clear that even the time independent version of equations (1.1)-(1.3) cannot be solved explicitly. Therefore, to establish conditions which guarantee the existence of a positive steady state we utilise a combination of an operator theoretic framework (see e.g. [5, 15]) and a fixed point approach. For basic definitions and results from linear semigroup theory used throughout this section we refer the reader to $[2,10,13]$.

For a fixed non vanishing $\mathbf{v} \in L_{+}^{1}(0, m) \oplus \mathbb{R}$ let us define the linear operator $\Psi_{\mathbf{v}}$ (parametrised by $\mathbf{v}$ ) by

$$
\Psi_{\mathbf{v}} \mathbf{u}=A \mathbf{u}+F_{\mathbf{v}}^{0} \mathbf{u},
$$

where the operator $A$ is defined in (2.6), and

$$
F_{\mathbf{v}}^{0} \mathbf{u}=\left(\begin{array}{c}
\beta_{0}(\|\mathbf{v}\|) \int_{0}^{m} \beta_{1}(\cdot, y) \beta_{2}\left(\frac{\int_{y}^{m} v(r) \mathrm{d} r}{\|\mathbf{v}\|}\right) u(y) \mathrm{d} y \\
\beta_{0}(\|\mathbf{v}\|) \int_{0}^{m} \beta_{1}(0, y) \beta_{2}\left(\frac{\int_{y}^{m} v(r) \mathrm{d} r}{\|\mathbf{v}\|}\right) u(y) \mathrm{d} y+b_{0}(\|\mathbf{v}\|) b_{2}\left(\frac{\int_{0}^{m} v(y) \mathrm{d} y}{\|\mathbf{v}\|}\right) u_{0}
\end{array}\right),
$$


with domain

$$
\begin{aligned}
D\left(\Psi_{\mathbf{v}}\right)= & \left\{u \in C^{2}([0, m]): \mathcal{A} u \in L^{1}(0, m), \quad d(m) u^{\prime}(m)-\gamma(m) u(m)=0\right. \\
& \left.\left.\left(d(s) u^{\prime}(s)\right)^{\prime}\right|_{s=0}-(d(0)+\gamma(0)) u^{\prime}(0)+\left(\gamma(0)-\gamma^{\prime}(0)\right) u(0)=0\right\} .
\end{aligned}
$$

It is clear that $\left(v_{*}, v_{*}(0)\right)$ is a nontrivial steady state of model (1.1)-(1.3) if (and only if) it is a positive eigenvector belonging to the kernel of the (closure of the) linear operator $\Psi_{\mathrm{v}}$.

Next we establish some (necessary) regularity properties of the semigroup generated by the closure of $\Psi_{\mathbf{v}}$. Several characterisations of irreducibility of a positive semigroup exist in the literature, see for example in [2, 10, 13]. Here we follow [13]. A positive semigroup $\mathcal{T}$ on the Banach lattice $\mathcal{X}$ is said to be irreducible if the resolvent of its generator $G$ is strictly positive, i.e. $\forall 0 \not \equiv f \in \mathcal{X}_{+}$we have $(R(\lambda, G) f)(x)>0$ for almost all $x$ and some $\lambda>s(G)$.

Lemma 3.2. For every fixed $\mathbf{v} \in \mathcal{X}_{+}$the closure of the linear operator $\Psi_{\mathbf{v}}$ generates an irreducible semigroup on $\mathcal{X}$.

Proof. We introduce the mortality operator $M$ as follows:

$$
M \mathbf{u}=\left(\begin{array}{c}
-\mu(\cdot) u \\
-\mu(0) u(0)
\end{array}\right) \quad \text { on } \quad \mathcal{X}_{+} \cdot
$$

We then consider the resolvent equation

$$
(\lambda \mathcal{I}-(A-M)) \mathbf{u}=\mathbf{h},
$$

for $\mathbf{h} \in \mathcal{X}_{+}, \lambda>0$, i.e.

$$
\begin{aligned}
-h(x) & =\left(d(x) u_{x}(x)-\gamma(x) u(x)\right)_{x}-\lambda u(x), \quad x \in(0, m), \\
-h_{0} & =-(\gamma(0)+\lambda) u(0)+d(0) u^{\prime}(0),
\end{aligned}
$$

for an unknown $u \in D(A)$. We have shown in [17, Theorem 2.1] that the linear semigroup generated by $A-M$ is positive (with two point Wentzell boundary conditions, but the proof can be trivially adapted to our case), i.e. the resolvent operator $(\lambda \mathcal{I}-(A-M))^{-1}$ is positive for $\lambda>0$ large enough. Hence the solution $\mathbf{u}$ of equations (3.21)-(3.22) is non-negative, i.e. $\mathbf{u} \geq 0$. It is only left to show that the solution $\mathbf{u}$ is in fact strictly positive.

The one-dimensional minimum principle assures that $u$ cannot attain its minimum value at an interior point of $(0, m)$, see e.g. [33, Ch. 1 Theorem 3]. On the other hand, [33, Ch. 1 Theorem 4] guarantees that if $u$ attains its non-positive minimum at 0 then $u^{\prime}(0)>0$ holds, which contradicts $\mathbf{h} \geq 0$, whereas the boundary condition at $x=m$ implies $u(m)>0$ by the same theorem. Therefore the semigroup generated by the closure of $A-M$ is irreducible. Since $M$ is a bounded multiplication operator and 
$F_{\mathbf{v}}^{0}$ is bounded and positive for every $\mathbf{v} \in \mathcal{X}_{+}$it follows that the closure of $\Psi_{\mathbf{v}}$ generates an irreducible semigroup for every $\mathbf{v} \in \mathcal{X}_{+}$, see e.g. [2, C-III Proposition 3.3].

Remark 3.3 First of all we note that every steady state $(v, v(0))$ of model (1.1)-(1.3) shall have regularity $W^{2,1}(0, m)$. This immediately implies that model (1.1)-(1.3) does not admit a steady state which has uninfected individuals only, i.e. of the form $(0, v(0))$, with $v(0) \neq 0$.

Secondly, as we noted before, a vector $\mathbf{v}=(v, v(0))$ is a steady state of (1.1)-(1.3) if and only if it is a positive eigenvector belonging to the kernel of the closure of $\Psi_{\mathbf{v}}$. Therefore, irreducibility of the semigroup generated by the closure of $\Psi_{\mathbf{v}}$ shows that we also cannot have a steady state of the form $(v, 0)$, since every positive eigenvector of the closure of the generator is strictly positive if the semigroup is irreducible.

Lemma 3.4. For every non vanishing $\mathbf{v} \in \mathcal{X}_{+}$the spectrum of $\Psi_{\mathbf{v}}$ can contain only isolated eigenvalues of finite algebraic multiplicity.

Proof. Since $M$ and $F_{\mathbf{v}}^{0}$ are bounded it is enough to show that $R(\lambda, A-M)$ is compact. This follows however by noting that the solution of the resolvent equation (3.21)-(3.22) is in $W^{2,1}(0, m) \oplus \mathbb{R}$ which is compactly embedded in $\mathcal{X}$. The statement now follows on the grounds of [13, Proposition II.4.25] and [13, Corollary IV.1.19].

As we noted before our goal is to show that there exists an element $\mathbf{v} \in \mathcal{X}_{+}$such that the operator $\Psi_{\mathbf{v}}$ has eigenvalue zero. Then, Lemmas 3.2 and 3.4 guarantee the existence of a corresponding strictly positive (unique normalized) eigenvector. In case of a model with one dimensional nonlinearity, such as the one we treated in [15], the operator $\Psi$ is in fact parametrized by a scalar quantity, namely, the total population size. In this case the positive steady state is obtained readily after an appropriate normalization of the positive eigenvector. In case of the model treated here the function $\beta_{2}$ naturally depends on an infinite dimensional variable. Therefore, in general there is no guarantee that the positive eigenvector corresponding to the zero eigenvalue of the operator $\Psi_{\mathbf{v}}$ is in fact $\mathbf{v}$ (or a scalar multiple of it). For this reason, we need to construct an appropriate nonlinear map on a certain level set of the positive cone of the state space $\mathcal{X}$ and establish the existence of a fixed point of this map. We note that a different fixed point strategy was employed very recently in [18] for a class of models with infinite dimensional nonlinearities (and zero flux boundary condition). That method, which uses fixed point results of nonlinear maps in conical shells of Banach spaces, does not apply to our model here since the construction of the nonlinear map requires the (implicit) solution of the steady state equation. We also note that a similar argument to the one used here, even though in the case of finite dimensional interaction variable is used in [7].

Theorem 3.5. Assume that $\beta_{0}$ and $b_{0}$ are strictly monotone decreasing functions of their argument and

$$
\text { (i) } \lim _{x \rightarrow \infty} \beta_{0}(x)=\lim _{x \rightarrow \infty} b_{0}(x)=0 \quad \text { and } \quad \mu(x)>\mu_{0}>0, \quad \forall x \in(0, m) \text {; }
$$


(ii) there exists an $r>0$ such that for all $\mathbf{v}_{*} \in \mathcal{X}_{+},\left\|\mathbf{v}_{*}\right\| \leq r$ we have that the spectral bound $s\left(\Psi_{\mathbf{v}_{*}}\right)>0$.

Then model (1.1)-(1.3) has at least one non-trivial steady state.

Proof. Since $\Psi_{\mathbf{v}}$ is a generator of a positive and irreducible semigroup with compact resolvent it follows that the spectrum $\sigma\left(\Psi_{\mathbf{v}}\right)$ is not empty, see e.g. [2, C-III Theorem 3.7]. Moreover, the spectral bound $s\left(\Psi_{\mathbf{v}}\right)$ is an isolated eigenvalue of algebraic multiplicity one with a corresponding strictly positive eigenvector and it is the unique eigenvalue with positive eigenvector, see e.g. [10, Theorem 9.10]. We also note that the spectral bound and the corresponding positive eigenvector change continuously with respect to (the parameter) v, see e.g. [30, Sect. 3 in Ch.4].

We introduce the level set

$$
S=\left\{\mathbf{x} \in \mathcal{X}_{+} \mid s\left(\Psi_{\mathbf{x}}\right)=0\right\} .
$$

It is shown that the closure of the linear operator $A-M$ generates a (positive) contraction semigroup, hence we have $s(A-M)=\omega_{0} \leq 0$. Intuitively it is clear that contractivity follows simply because in the absence of mortality and recruitment the total population size is preserved. Therefore, if

$$
\left\|F_{\mathbf{v}}^{0}\right\|<\inf _{x \in[0, m]}\{\mu(x)\},
$$

then it is shown that $s\left(\Psi_{\mathbf{v}}\right)<0$. Hence conditions (i) imply that there exists an $R>0$ such that for $\forall \mathbf{v}_{*} \in \mathcal{X}_{+},\left\|\mathbf{v}_{*}\right\| \geq R$ we have $s\left(\Psi_{\mathbf{v}_{*}}\right)<0$. From (ii) it then follows that $S \subset\left\{\mathbf{x} \in \mathcal{X}_{+} \mid r<\|\mathbf{x}\|<R\right\}$. It also follows from conditions (i) and (ii) and from the continuity and strict monotonicity of the spectral bound (see below) that along every positive ray $\mathcal{R}=\left\{\alpha \mathbf{v}_{*} \mid \alpha \in \mathbb{R}_{+}, \mathbf{v}_{*} \in \mathcal{X}_{+} \backslash\{\mathbf{0}\}\right\}$ there exists a (unique!) $\mathbf{v}$ such that $s\left(\Psi_{\mathbf{v}}\right)=0$. So the set $S$ intersects every positive ray $\mathcal{R}$ in a unique element.

Next, we shall show that the spectral bound is in fact strictly monotone decreasing along every positive ray. To this end let $0<\alpha_{1}<\alpha_{2}$ be real numbers and let $\mathbf{v} \in \mathcal{X}_{+}$. Consider the operators $\Psi_{\alpha_{1} \mathbf{v}}$ and $\Psi_{\alpha_{2} \mathbf{v}}$. Both of them have compact resolvents by Lemma 3.4 and generate positive and irreducible semigroups. We also note that $\Psi_{\alpha_{1} \mathbf{v}}-\Psi_{\alpha_{2} \mathbf{v}}$ is a positive operator, since

$$
\begin{aligned}
& \left(\Psi_{\alpha_{1} \mathbf{v}}-\Psi_{\alpha_{2} \mathbf{v}}\right) \mathbf{u}=\left(F_{\alpha_{1} \mathbf{v}}^{0}-F_{\alpha_{2} \mathbf{v}}^{0}\right) \mathbf{u} \\
& =\left(\begin{array}{c}
\left(\beta_{0}\left(\alpha_{1}\|\mathbf{v}\|\right)-\beta_{0}\left(\alpha_{2}\|\mathbf{v}\|\right)\right) \int_{0}^{m} \beta_{1}(\cdot, y) \beta_{2}\left(\frac{\int_{y}^{m} v(r) \mathrm{d} r}{\|\mathbf{v}\|}\right) u(y) \mathrm{d} y \\
\left(\beta_{0}\left(\alpha_{1}\|\mathbf{v}\|\right)-\beta_{0}\left(\alpha_{2}\|\mathbf{v}\|\right)\right) \int_{0}^{m} \beta_{1}(0, y) \beta_{2}\left(\frac{\int_{y}^{m} v(r) \mathrm{d} r}{\|\mathbf{v}\|}\right) u(y) \mathrm{d} y
\end{array}\right) \\
& +\left(\begin{array}{c}
0 \\
\left(b_{0}\left(\alpha_{1}\|\mathbf{v}\|\right)-b_{0}\left(\alpha_{2}\|\mathbf{v}\|\right)\right) b_{2}\left(\frac{\int_{0}^{m} v(y) \mathrm{d} y}{\|\mathbf{v}\|}\right) u_{0}
\end{array}\right) \\
& \geq 0 \text {, }
\end{aligned}
$$


for every $\mathbf{u} \in \mathcal{X}_{+}$. We also have

$$
R\left(\lambda, \Psi_{\alpha_{1} \mathbf{v}}\right)-R\left(\lambda, \Psi_{\alpha_{2} \mathbf{v}}\right)=R\left(\lambda, \Psi_{\alpha_{1} \mathbf{v}}\right)\left(\Psi_{\alpha_{1} \mathbf{v}}-\Psi_{\alpha_{2} \mathbf{v}}\right) R\left(\lambda, \Psi_{\alpha_{2} \mathbf{v}}\right) \geq 0,
$$

for $\lambda$ large enough. Hence [1, Proposition A.2] implies that $s\left(\Psi_{\alpha_{1} \mathbf{v}}\right)>s\left(\Psi_{\alpha_{2} \mathbf{v}}\right)$.

Next we construct the nonlinear map $\Phi: S \rightarrow S, \Phi(\mathbf{v})=\left(f_{4} \circ f_{3} \circ f_{2} \circ f_{1}\right)(\mathbf{v})=\mathbf{v}^{\prime}$, as illustrated briefly in the following diagram:

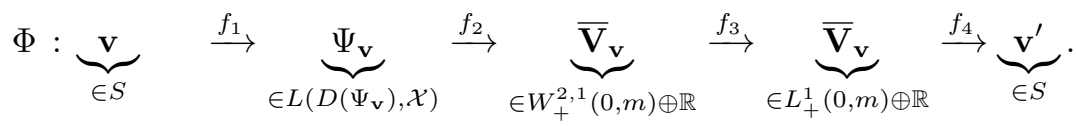

For every element $\mathbf{v} \in S$ the map $f_{1}$ assigns the corresponding linear operator $\Psi_{\mathbf{v}}$ "parametrized" by $\mathbf{v}$, which is defined via (3.17). This map $f_{1}$ is continuous and bounded. The map $f_{2}$ assigns the strictly positive normalized eigenvector corresponding to the zero eigenvalue of the operator $\Psi_{\mathbf{v}}$. This map $f_{2}$ is clearly bounded and it is also continuous, in fact it is even analytic, see [11, Lemma 1.3]. The map $f_{3}$ is the compact injection of $W^{2,1}(0, m) \oplus \mathbb{R}$ into $L^{1}(0, m) \oplus \mathbb{R}$. Finally $f_{4}$ is the projection along positive rays of the eigenvector $\overline{\mathbf{V}}_{\mathbf{v}}$ back into the set $S$, which is again continuous and bounded.

Next we apply Schauder's fixed point theorem to an appropriately defined map. As we noted above the map $\Phi$ is continuous and compact. It is only left to show that $S$ is homeomorphic to a convex set. To this end, we define the map $h: S \rightarrow B$ by $h(\mathbf{u})=\frac{\mathbf{u}}{\|\mathbf{u}\|}$, where $B$ is the unit sphere intersected with the positive cone $\mathcal{X}_{+}$, i.e.

$$
B:=\left\{\mathbf{u}=\left(u, u^{0}\right) \in \mathcal{X}_{+}\left|\|\mathbf{u}\|=\|u\|_{1}+\right| u^{0} \mid=1\right\} .
$$

Then $h$ is clearly continuous and one to one, since the spectral bound $s(\Psi)$ is strictly monotone decreasing along positive rays in $\mathcal{X}$ and every positive ray of $\mathcal{X}$ intersects $B$ in a unique element.

We shall show now that the function $h^{-1}: B \rightarrow S$ defined via $h^{-1}(\mathbf{w})=\alpha \mathbf{w}$, $\alpha \in \mathbb{R}_{+}$such that $s\left(\Psi_{\alpha \mathbf{w}}\right)=0$, is also continuous. Let $\mathbf{w}_{n} \in B$ be a sequence such that $\mathbf{w}_{n} \rightarrow \mathbf{w} \in B$ and consider the sequence $h^{-1}\left(\mathbf{w}_{n}\right)=\alpha_{n} \mathbf{w}_{n}$. It follows from condition (i) that there exists an $R>0$ such that $s\left(\Psi_{\mathbf{v}}\right)<0$ for every $\|\mathbf{v}\|>R$, hence the sequence $\alpha_{n}$ is bounded. Let $\alpha_{n_{k}}$ be a convergent subsequence of $\alpha_{n}$ and let $\alpha_{n_{k}} \rightarrow \bar{\alpha}$. Since $s\left(\Psi_{\alpha_{n_{k}} \mathbf{w}_{n_{k}}}\right)=0$ for every $k \in \mathbb{N}$ it follows from the continuity of the spectral bound that $s\left(\Psi_{\bar{\alpha} \mathbf{w}}\right)=0$. Since there is exactly one element on the positive ray spanned by w at which the spectral bound vanishes we have $\bar{\alpha}=\alpha$. If $\alpha_{n_{l}}$ is another convergent subsequence of $\alpha_{n}$ then the continuity of the spectral bound implies again that $\alpha_{n_{l}} \rightarrow \alpha$. So $\alpha_{n} \rightarrow \alpha$ and finally $h^{-1}\left(\mathbf{w}_{n}\right)=\alpha_{n} \mathbf{w}_{n} \rightarrow \alpha \mathbf{w}=h^{-1}(\mathbf{w})$.

We note that the set $B$ is convex since we are in an AL-space, i.e. we have $\|\mathbf{f}+\mathbf{g}\|=$ $\|\mathbf{f}\|+\|\mathbf{g}\|$ for every $\mathbf{f}, \mathbf{g} \in \mathcal{X}_{+}$, see e.g. [2]. Finally we apply Schauder's fixed point theorem (see e.g. [24]) to the continuous and compact map $\bar{\Phi}: B \rightarrow B$ defined by

$$
\bar{\Phi}(\mathbf{x})=h \circ \Phi \circ h^{-1}(\mathbf{x}),
$$


to obtain a fixed point $\overline{\mathbf{x}}_{*} \in B$ of the map $\bar{\Phi}$, which yields a fixed point $\mathbf{x}_{*}=h^{-1}\left(\overline{\mathbf{x}}_{*}\right)$ of the map $\Phi$ in $S$. This $\mathbf{x}_{*}$ is the positive steady state of model (1.1)-(1.3) (see the characterization of a nontrivial steady state immediately after equation (3.18)).

Remark 3.6 Condition (i) is natural from the biological point of view. The first condition in (i) requires that the fertility rate of both infected and uninfected individuals tends to zero as the population size tends to infinity. This may be due for example to competition effects. In fact it turns out that as expected, cytoplasmic incompatibility itself does not have a negative feedback on population growth. The assumption of a strictly positive mortality function in (i) seems also realistic.

Condition (ii) seems to be also a natural one, if one is to expect the existence of a positive steady state. In fact, if it is not satisfied then one can show that there exists a monotone decreasing sequence of positive real numbers $r_{n} \rightarrow 0$, such that for every $n \in \mathbb{N}$ there exists a $\mathbf{u}_{*}^{n}$ with $\left\|\mathbf{u}_{*}^{n}\right\| \leq r_{n}$ and $s\left(\Psi_{\mathbf{u}_{*}^{n}}\right) \leq 0$ holds. Then, the continuity of the spectral bound implies that $s\left(\Psi_{\mathbf{0}}\right) \leq 0$ and since the spectral bound is a strictly monotone decreasing function along positive rays, we have $s\left(\Psi_{\mathbf{v}}\right) \leq 0$, for every $\mathbf{v} \in \mathcal{X}_{+}$.

\section{Stability}

In the previous section we established conditions which guarantee the existence of a non-trivial steady state. The next natural step is to study the stability of the steady state (and also the stability of the trivial steady state). In this section we are going to establish a sufficient condition for the local asymptotic stability of the non-trivial steady state. First we note that the operator $F$ is not (Fréchet) differentiable at $\mathbf{0}$ because it is homogeneous of degree 1 (it is an "angular operator"). Nevertheless it is Gâteauxdifferentiable at $\mathbf{0}$. In particular its directional derivative at $\mathbf{0}$ into the direction of any element $\mathbf{v}$ in the positive cone is:

$$
\mathrm{d} F_{\mathbf{0}} \mathbf{v}=\left(\begin{array}{c}
\beta_{0}(0) \int_{0}^{m} \beta_{1}(\cdot, y) \beta_{2}\left(\frac{\int_{y}^{m} v(r) \mathrm{d} r}{\|\mathbf{v}\|}\right) v(y) \mathrm{d} y \\
\beta_{0}(0) \int_{0}^{m} \beta_{1}(0, y) \beta_{2}\left(\frac{\int_{y}^{m} v(r) \mathrm{d} r}{\|\mathbf{v}\|}\right) v(y) \mathrm{d} y+b_{0}(0) b_{2}\left(\frac{\int_{0}^{m} v(y) \mathrm{d} y}{\|\mathbf{v}\|}\right) v_{0}
\end{array}\right),
$$

which is a nonlinear operator.

If $\mathbf{u}_{*}$ is a non-trivial equilibrium of (1.1)-(1.3) then we can formally linearise equation (1.1) around $\mathbf{u}_{*}$. In particular if we denote by $F_{\mathbf{u}_{*}}^{\prime}$ the linearisation (the Fréchet derivative) of the nonlinear operator $F$ at $\mathbf{u}_{*}$ then the linearised problem can be cast in the form of an abstract Cauchy problem

$$
\frac{\mathrm{d}}{\mathrm{d} t} \mathbf{v}=\left(A+F_{\mathbf{u}_{*}}^{\prime}\right) \mathbf{v}, \quad \mathbf{v}(0)=\mathbf{v}_{0}
$$

where $A$ is defined in (2.6) and $D\left(F_{\mathbf{u}_{*}}^{\prime}\right)=\mathcal{X}$. We leave it for future work to address the linear problem (4.24) (i.e. to address stability questions of equilibria), in general, for example using the Liapunov function techniques elaborated in [38, Sect. 4.2]. Here 
however, we establish a straightforward and simple condition which guarantees that the non-trivial equilibrium is locally asymptotically stable.

To this end we consider on the state space $\mathcal{X}$ the operator $F$ defined in (2.8) with domain $D(F)=\mathcal{X}^{+}$. Then using approximations (in the spirit of [38, Def.2.4 in Sect. 2.6]) such as

$$
\beta_{0}\left(\left\|\mathbf{u}_{*}+\mathbf{v}\right\|\right) \sim \beta_{0}\left(\left\|\mathbf{u}_{*}\right\|\right)+\beta_{0}^{\prime}\left(\left\|\mathbf{u}_{*}\right\|\right)\left(\int_{0}^{m} v(y) \mathrm{d} y+v_{0}\right)
$$

and similarly

$$
\begin{aligned}
\beta_{2}\left(\frac{\int_{y}^{m} u_{*}(r)+v(r) d r}{\left\|\mathbf{u}_{*}+\mathbf{v}\right\|}\right) & \sim \beta_{2}\left(\frac{\int_{y}^{m} u_{*}(r) d r}{\left\|\mathbf{u}_{*}\right\|}\right) \\
& +\beta_{2}^{\prime}\left(\frac{\int_{y}^{m} u_{*}(r) d r}{\left\|\mathbf{u}_{*}\right\|}\right)\left(\frac{\int_{y}^{m} v(r) d r}{\left\|\mathbf{u}_{*}\right\|}-\int_{y}^{m} u_{*}(r) d r \frac{\int_{0}^{m} v(y) d y+v_{0}}{\left\|\mathbf{u}_{*}\right\|^{2}}\right)
\end{aligned}
$$

it is shown that the linearisation of the operator $F$ at the non-trivial equilibrium $\mathbf{u}_{*}$ (according again to [38, Def. 2.4 in Sect. 2.6] is

$$
\begin{aligned}
& F_{\mathbf{u}_{*} \mathbf{v}=}^{\prime} \\
& \left(\begin{array}{l}
\int_{0}^{m} \beta_{1}(\cdot, y) \beta_{2}\left(\frac{\int_{y}^{m} u_{*}(r) d r}{\left\|\mathbf{u}_{*}\right\|}\right)\left(\beta_{0}\left(\left\|\mathbf{u}_{*}\right\|\right) v(y)+\beta_{0}^{\prime}\left(\left\|\mathbf{u}_{*}\right\|\right)\left(\int_{0}^{m} v(y) d y+v_{0}\right) u_{*}(y)\right) \mathrm{d} y \\
\int_{0}^{m} \beta_{1}(0, y) \beta_{2}\left(\frac{\int_{y}^{m} u_{*}(r) d r}{\left\|\mathbf{u}_{*}\right\|}\right)\left(\beta_{0}\left(\left\|\mathbf{u}_{*}\right\|\right) v(y)+\beta_{0}^{\prime}\left(\left\|\mathbf{u}_{*}\right\|\right)\left(\int_{0}^{m} v(y) d y+v_{0}\right) u_{*}(y)\right) \mathrm{d} y
\end{array}\right) \\
+ & \left(\begin{array}{l}
\int_{0}^{m} \beta_{1}(\cdot, y) \beta_{0}\left(\left\|\mathbf{u}_{*}\right\|\right) \beta_{2}^{\prime}\left(\frac{\int_{y}^{m} u_{*}(r) d r}{\left\|\mathbf{u}_{*}\right\|}\right)\left(\frac{\int_{y}^{m} v(r) d r}{\left\|\mathbf{u}_{*}\right\|}-\int_{y}^{m} u_{*}(r) d r \frac{\int_{0}^{m} v(y) d y+v_{0}}{\left\|\mathbf{u}_{*}\right\|^{2}}\right) u_{*}(y) \mathrm{d} y \\
\int_{0}^{m} \beta_{1}(0, y) \beta_{0}\left(\left\|\mathbf{u}_{*}\right\|\right) \beta_{2}^{\prime}\left(\frac{\int_{y}^{m} u_{*}(r) d r}{\left\|\mathbf{u}_{*}\right\|}\right)\left(\frac{\int_{y}^{m} v(r) d r}{\left\|\mathbf{u}_{*}\right\|}-\int_{y}^{m} u_{*}(r) d r \frac{\int_{0}^{m} v(y) d y+v_{0}}{\left\|\mathbf{u}_{*}\right\|^{2}}\right) u_{*}(y) \mathrm{d} y
\end{array}\right) \\
+ & \left(\begin{array}{l}
b_{2}\left(\frac{\int_{0}^{m} u_{*}(y) d y}{\left\|\mathbf{u}_{*}\right\|}\right)\left[b_{0}\left(\left\|\mathbf{u}_{*}\right\|\right) v_{0}+b_{0}^{\prime}\left(\left\|\mathbf{u}_{*}\right\|\right)\left(\int_{0}^{m} v(y) d y+v_{0}\right) u_{*_{0}}\right]
\end{array}\right) \\
+ & \left(\begin{array}{c}
0 \\
b_{0}\left(\left\|\mathbf{u}_{*}\right\|\right) b_{2}^{\prime}\left(\frac{\int_{0}^{m} u_{*}(y) d y}{\left\|\mathbf{u}_{*}\right\|}\right)\left(\frac{\int_{0}^{m} v(y) d y}{\left\|\mathbf{u}_{*}\right\|}-\frac{\int_{0}^{m} u_{*}(y) d y}{\left\|\mathbf{u}_{*}\right\|^{2}}\left(\int_{0}^{m} v(y) d y+v_{0}\right)\right) u_{*_{0}}
\end{array}\right),
\end{aligned}
$$

which is a bounded linear operator on $\mathcal{X}$.

Theorem 4.7. If

$$
\nu:=\inf _{s \in[0, m]}\{\mu(s)\}>\left\|F_{\mathbf{u}_{*}}^{\prime}\right\|,
$$

then the non-trivial steady state $\mathbf{u}_{*}$ is locally asymptotically stable.

Proof. Note that $A-M$ has compact resolvent (where $M$ is the mortality operator introduced in (3.19)), see the proof of Lemma 3.4, and generates a positive and irreducible semigroup, see the proof of Lemma 3.2. Therefore its point spectrum $\sigma_{P}(A-M)$ is not empty, see [2, C-III Theorem 3.7]. We also noted before that it generates a contraction semigroup. In fact, the semigroup $\mathcal{T}_{1}(t)$ generated by $A-M$ satisfies $\left\|\mathcal{T}_{1}(t)\right\|_{\mathcal{X}}=1$ 
for every $t>0$, hence $0=\omega_{0}(A-M)=s(A-M)$. On the other hand we have $\left\|\exp \left\{t F_{\mathbf{u}_{*}}^{\prime}\right\}\right\| \leq \exp \left\{t\left\|F_{\mathbf{u}_{*}}^{\prime}\right\|\right\}$, hence for the growth bound of the semigroup $\mathcal{T}_{2}(t)$ generated by $F_{\mathbf{u}_{*}}^{\prime}$ we have $\omega_{0}\left(F_{\mathbf{u}_{*}}^{\prime}\right) \leq\left\|F_{\mathbf{u}_{*}}^{\prime}\right\|$. We also note that $M+\nu \mathcal{I}$ is a dissipative operator and it generates a contraction semigroup, hence for the growth bound of the semigroup $\mathcal{T}_{3}(t)$ generated by $M$ we have $\omega_{0}(M)<-\nu$, and $\left\|\mathcal{T}_{3}(t)\right\| \leq \exp \{-\nu t\}$. Finally, by applying a version of the Trotter product formula (see e.g. [13, Corollary 5.8]) we obtain $\omega_{0}\left(A-M+F_{\mathbf{u}_{*}}^{\prime}+M\right)=\omega_{0}\left(A+F_{\mathbf{u}_{*}}^{\prime}\right)<0$ which shows that the nontrivial steady state $\mathbf{u}_{*}$ is locally asymptotically stable.

Remark 4.8 The stability condition in Theorem 4.7 may seem very restrictive at the first glance. It may however be the case that the norm of the linearisation $\left\|F_{\mathbf{u}_{*}}^{\prime}\right\|$ is small, especially since for our model we naturally have $\beta_{0}^{\prime}, b_{0}^{\prime}, \beta_{2}^{\prime}, b_{2}^{\prime} \leq 0$. On the other hand, even natural mortality (note that $\mu$ is natural mortality combined with infection induced mortality) is very high for example in the case of the mosquito Aedes aegypti, which is one of the target species for the introduction of Wolbachia, see [31]. We also note that the operator $-F_{\mathbf{u}_{*}}^{\prime}$ may well be positive if the conditions of Theorem 3.5 are satisfied, but there are no readily available results concerning the question that how does the infimum of the spectrum of an operator changes under positive perturbations. On the other hand if $F_{\mathbf{u}_{*}}^{\prime}$ is dissipative and $\nu>0$ then similar arguments as used in the proof of Theorem 4.7 show that the steady state is locally asymptotically stable.

Remark 4.9 As we pointed out earlier, it is not possible to talk about linear stability of the trivial steady state in the usual sense, as the nonlinearity $F$ is not Fréchet differentiable at $\mathbf{0}$. We established however global existence of solutions for any initial condition, and since condition (ii) in Theorem 3.5 requires that the spectral bound of the linear part is positive in a small neighbourhood of $\mathbf{0}$ it may be intuitively plausible to expect (for example utilising the variation formula (2.16)) that the trivial steady state is unstable and the positive steady state is actually globally asymptotically stable if the condition of Theorem 4.7 holds true.

\section{CONCLUding REMARKS}

In this paper we introduced and analysed a nonlinear structured population model with diffusion in the state space. Individuals in the population are structured with respect to infection (for example bacterium) load, hence we used Wentzell boundary condition at the uninfected state $x=0$. Our model primarily intended to describe the evolution of Wolbachia infection in an arthropod, for example mosquito population. Wolbachia is a reproductive parasite and it affects the reproductive mechanisms of its host in an intriguing fashion. Here we focused on a cytoplasmic incompatibility (CI) inducing strain. Following $[6,25]$ we adopted the view of partial CI, namely, that a female can produce viable offspring only when mating with a male who has lower infection load. Therefore the functions $\beta_{2}, b_{2}$ are assumed to be monotone decreasing. Note however, that these assumptions are not necessary to establish any of our results, such as Theorem 2.1 and 
Theorem 3.5. In fact, in our main result Theorem 3.5, the crucial assumption is the one which concerns the strict monotonocity of the functions $\beta_{0}, b_{0}$. The necessity of condition (i) in Theorem 3.5 is also in agreement with the fact that CI itself does not regulate population growth, it only provides infected individuals with a reproductive advantage, see e.g. [14, 16, 34]. The main difficulty in the mathematical analysis of our model also arises from this crucial assumption of the density dependent CI. In particular, physiologically structured population models with infinite dimensional nonlinearities and with distributed recruitment terms, such as the one used here, are usually notoriously difficult to analyse, see e.g. $[8,18]$. The special form of the nonlinearity naturally appearing in $\beta_{2}$ and $b_{2}$ also implies that although the nonlinear operator $F$ is locally Lipschitz continuous at $\mathbf{0}$ it is not Fréchet differentiable. This also implies that even though intuitively one may expect that if the conditions of Theorem 3.5 and Theorem 4.7 hold true then the trivial steady state is unstable, we cannot discuss linear stability of the trivial steady state using standard stability results from semilinear theory as developed for example in $[28,38]$.

\section{ACKNOWLEDGMENTS}

À. Calsina was partially supported by the research projects DGI MTM2008-06349C03-03 and 2009SGR-345. J. Z. Farkas was supported by a University of Stirling Research and Enterprise Support Grant and a Royal Society of Edinburgh International Travel Grant.

\section{REFERENCES}

[1] W. Arendt and C.J.K Batty, Principal eigenvalues and perturbation, Oper. Theory Adv. Appl. 75 (1995), 39-55.

[2] W. Arendt, A. Grabosch, G. Greiner, U. Groh, H. P. Lotz, U. Moustakas, R. Nagel, F. Neubrander and U. Schlotterbeck, One-Parameter Semigroups of Positive Operators, Springer-Verlag, Berlin, (1986).

[3] H. T. Banks, J. L. Davis, S. L. Ernstberger, S. Hu, E. Artimovich, A. K. Dhar and C. L. Browdy, A comparison of probabilistic and stochastic formulations in modelling growth uncertainty and variability, J. Biol. Dyn. 3 (2009), 130-148.

[4] H. T. Banks, J. L. Davis and S. Hu, A computational comparison of alternatives to including uncertainty in structured population models, Three Decades of Progress in Control Sciences, SpringerVerlag, (2010), 19-33.

[5] R. Borges, À. Calsina, S. Cuadrado, Equilibria of a cyclin structured cell population model, Discrete Contin. Dyn. Syst., Ser. B 11 (2009), 613-627.

[6] K. Bourtzis, A. Nirgianaki, G. Markakis and C. Savakis, Wolbachia infection and cytoplasmic incompatibility in Drosophila species, Genetics 144 (1996), 1063-1073.

[7] À. Calsina and J. M. Palmada, Steady states of a selection-mutation model for an age structured population, submitted.

[8] À. Calsina and J. Saldaña, Basic theory for a class of models of hierarchically structured population dynamics with distributed states in the recruitment, Math. Models Methods Appl. Sci. 16 (2006), $1695-1722$ 
[9] J. Chu, A. Ducrot, P. Magal and S. Ruan, Hopf bifurcation in a size-structured population dynamic model with random growth, J. Differential Equations 247 (2009), 956-1000.

[10] Ph. Clément, H. J. A. M Heijmans, S. Angenent, C. J. van Duijn, and B. de Pagter, One-Parameter Semigroups, North-Holland, Amsterdam (1987).

[11] M. G. Crandall and P. H. Rabinowitz, Bifurcation, perturbation of simple eigenvalues and linearized stability, Arch. Rational Mech. Anal., 52 (1973), 161-180.

[12] J. M. Cushing, An Introduction to Structured Population Dynamics, SIAM, Philadelphia, (1998).

[13] K.-J. Engel and R. Nagel, One-Parameter Semigroups for Linear Evolution Equations, Springer, New York 2000.

[14] J. Engelstädter, A. Telschow and P. Hammerstein, Infection dynamics of different Wolbachia-types within one host population, J. Theoret. Biol. 231 (2004), 345-355.

[15] J. Z. Farkas, D. Green and P. Hinow, Semigroup analysis of structured parasite populations, Math. Model. Nat. Phenom., 8 (2010), 94-114.

[16] J. Z. Farkas and P. Hinow, Structured and unstructured continuous models for Wolbachia infections, Bull. Math. Biol., 72 (2010), 2067-2088.

[17] J. Z. Farkas and P. Hinow, Physiologically structured populations with diffusion and dynamic boundary conditions, Math. Biosci. Eng., 14 (2011), 503-513.

[18] J. Z. Farkas and P. Hinow, Steady states in hierarchical structured populations with distributed states at birth, to appear in Discrete Contin. Dyn. Syst., Ser. B.

[19] A. Favini, G. R. Goldstein, J. A. Goldstein and S. Romanelli, $C_{0}$-semigroups generated by second order differential operators with general Wentzell boundary conditions, Proc. Amer. Math. Soc. 128 (2000), 1981-1989.

[20] A. Favini, G. R. Goldstein, J. A. Goldstein and S. Romanelli, The heat equation with generalized Wentzell boundary condition, J. Evol. Equ. 2 (2002), 1-19.

[21] A. Favini, G. R. Goldstein, J. A. Goldstein, E. Obrecht and S. Romanelli, Elliptic operators with general Wentzell boundary conditions, analytic semigroups and the angle concavity theorem, Math. Nachr. 283 (2010), 504-521.

[22] W. Feller, The parabolic differential equations and the associated semi-groups of transformations, Ann. of Math. 55 (1952), 468-519.

[23] W. Feller, Diffusion processes in one dimension, Trans. Amer. Math. Soc. 77 (1954), 1-31.

[24] D. Gilbarg and N. Trudinger, Elliptic Partial Differential Equations of Second Order, SpringerVerlag, Berlin, (1983).

[25] T. Guillemaud and F. Rousset, Consequences of Wolbachia transmission process on the infection dynamics, J. Evol. Biol. 10 (1997), 601-612.

[26] G. R. Goldstein, Derivation and physical interpretation of general boundary conditions, Adv. Differential Equations 11 (2006), 457-480.

[27] K. P. Hadeler, Structured populations with diffusion in state space, Math. Biosci. Eng. 7 (2010), 37-49.

[28] D. Henry, Geometric theory of semilinear parabolic equations, Springer, Berlin-New York, (1981).

[29] M. Iannelli, Mathematical Theory of Age-Structured Population Dynamics, Giardini Editori, Pisa, (1994).

[30] T. Kato, Perturbation Theory for Linear Operators, Springer, New York, (1966).

[31] C. J. McMeniman, et al., Stable introduction of a life-shortening Wolbachia infection into the mosquito Aedes aegypti, Science 323 (2009), 141-144.

[32] J. A. J. Metz and O. Diekmann, The Dynamics of Physiologically Structured Populations, Springer, Berlin, (1986).

[33] M. H. Protter and H. F. Weinberger, Maximum principles in differential equations, Prentice-Hall, (1967). 
[34] S. P. Sinkins, Wolbachia and cytoplasmic incompatibility in mosquitoes, Insect Biochem. Molec. Biol. 34 (2004), 723-729.

[35] A. D. Ventcel, Semigroups of operators that correspond to a generalized differential operator of second order (Russian), Dokl. Akad. Nauk SSSR (N.S.) 111 (1956), 269-272.

[36] A. D. Ventcel, On boundary conditions for multi-dimensional diffusion processes, Theor. Probability Appl. 4 (1959), 164-177.

[37] R. Waldstätter, K. P. Hadeler and G. Greiner, A Lotka-McKendrick model for a population structured by the level of parasitic infection, SIAM J. Math. Anal. 19 (1988), 1108-1118.

[38] G. F. Webb, Theory of Nonlinear Age-Dependent Population Dynamics, Marcel Dekker, New York, (1985)

Àngel Calsina, Department of Mathematics, Universitat Autònoma de Barcelona, BelLaterra, Spain

E-mail address: acalsina@mat.uab.es

József Z. Farkas, Institute of Computing Science and Mathematics, University of Stirling, Stirling, FK9 4LA, United Kingdom

E-mail address: jzf@maths.stir.ac.uk 